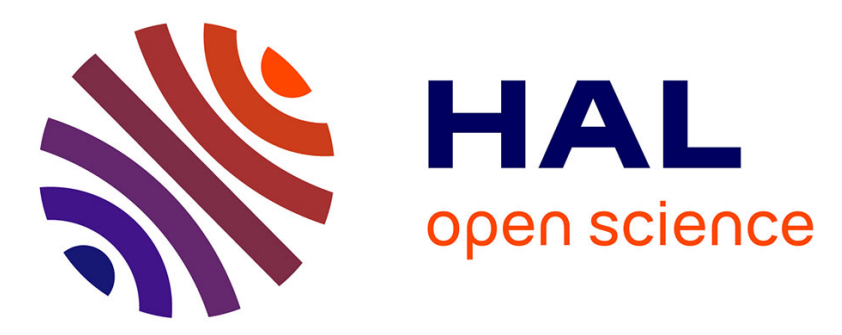

\title{
A comparison of wealth inequality in humans and non-humans
}

Ivan D Chase, Raphaël Douady, Dianna K Padilla

\section{To cite this version:}

Ivan D Chase, Raphaël Douady, Dianna K Padilla. A comparison of wealth inequality in humans and non-humans. Physica A: Statistical Mechanics and its Applications, 2020, 538, pp.122962. 10.1016/j.physa.2019.122962 . hal-03018472

\section{HAL Id: hal-03018472 \\ https://hal-paris1.archives-ouvertes.fr/hal-03018472}

Submitted on 22 Nov 2020

HAL is a multi-disciplinary open access archive for the deposit and dissemination of scientific research documents, whether they are published or not. The documents may come from teaching and research institutions in France or abroad, or from public or private research centers.
L'archive ouverte pluridisciplinaire HAL, est destinée au dépôt et à la diffusion de documents scientifiques de niveau recherche, publiés ou non, émanant des établissements d'enseignement et de recherche français ou étrangers, des laboratoires publics ou privés. 


\section{A Comparison of Wealth Inequality in Humans and Non-Humans}

Ivan D. Chase ${ }^{1, *}$, Raphael Douady ${ }^{2}$, Dianna K. Padilla ${ }^{3}$

${ }^{1}$ Department of Sociology and Graduate Program in Ecology and Evolution, Stony Brook University, Stony Brook, NY, USA

${ }^{2}$ Department of Applied Mathematics and Statistics, Stony Brook University, Stony Brook, NY, USA

${ }^{3}$ Department of Ecology and Evolution, Stony Brook University, Stony Brook, NY, USA

* Correspondence to: Department of Sociology, Stony Brook University, Stony Brook University, Stony Brook, NY 11794-4356, USA.

E-mail address: ivan.chase@stonybrook.edu.

\section{HIGHLIGHTS}

- We present the first description of "wealth" inequality in a non-human animal

- We describe the distribution of snail shells occupied by a hermit crab species

- The distribution of shells resembles the common form of human wealth distributions

- Hermit crabs may provide an animal model of the dynamics generating wealth inequality

- Shell distribution in hermit crabs provides a baseline to compare to human inequality

Keywords:

Wealth distribution

Power-law (Pareto) distributions

Fat tails

Vacancy chains

Hermit crabs

Human-animal comparisons

\section{ABSTRACT}

Inequality in the distribution of material resources (wealth) occurs widely across human groups. The extent of inequality, as measured by the Gini coefficient, is less in small-scale societies, such as hunter-gatherers and pastoralists, and greater in large-scale ones like current 
nation states. In many societies, the statistical distribution of wealth takes a characteristic form: unimodal, skewed to the right, and fat-tailed. However, we have relatively little systematic information about the distribution of material resources in nonhuman animals even though such resources are vital to their survival and fitness. Here we present the first description of inequality in material resources in an animal population: the distribution of gastropod (snail) shells inhabited by the hermit crab Pagurus longicarpus. We find that the shell distribution for the crabs strongly resembles the characteristic form of wealth distribution in human groups. The amount of inequality in the crabs is more than that in some small-scale human groups but less than that in nations. We argue that the shell distribution in the crabs is not simply generated by biological factors such as survival and growth of either crabs or gastropods. Instead, the strong resemblance in the human and hermit crab distributions suggests that comparable factors, not dependent upon culture or social institutions, could shape the patterns of inequality in both groups. In addition to the similarity in their inequality distributions, human and hermit crabs share other features of resource distribution, including the use of vacancy chains, not seen in other species. Based upon these parallels, we propose that $P$. longicarpus could be used as an animal model to test two factors - individual differences and intergenerational property transfers - that some economists theorize as major factors influencing the form of wealth distributions in humans. We also propose that inequality in hermit crabs could provide a baseline for examining human inequality.

\section{INTRODUCTION}

Inequality in material possessions, or wealth, occurs widely in human societies. Researchers have documented it in contemporary as well as historical societies, and in large- as well as small-scale groups $[1,2,3,4]$. However, there is little systematic information about the 
distribution of material possessions in nonhuman animals, even though things such as nests, dens, shelters, and territories are important to survival and fitness [5]. Here, we present, as far as we know, the first description of "wealth" inequality in an animal population: the statistical distribution of empty gastropod (snail) shells occupied by individuals of the hermit crab Pagurus longicarpus (but see [6] for a comparison of human wealth inequality and species abundance).We compare the distribution we find in the crabs with that in various human groups.

It might seem surprising to compare inequality in humans and hermit crabs since, in most respects, they are very different as species. Their evolutionary lines split over 550 million years ago, and hermit crabs are completely without culture and social institutions. However, human resources and snail shells as used by hermit crabs do share a number of abstract qualities. Resources in both species are often scarce, discrete, owned by identifiable individuals, and quantifiable in size or value. In addition, in many cases, both shells in hermit crabs and resources in humans are distributed through the process known as vacancy chains (defined below).

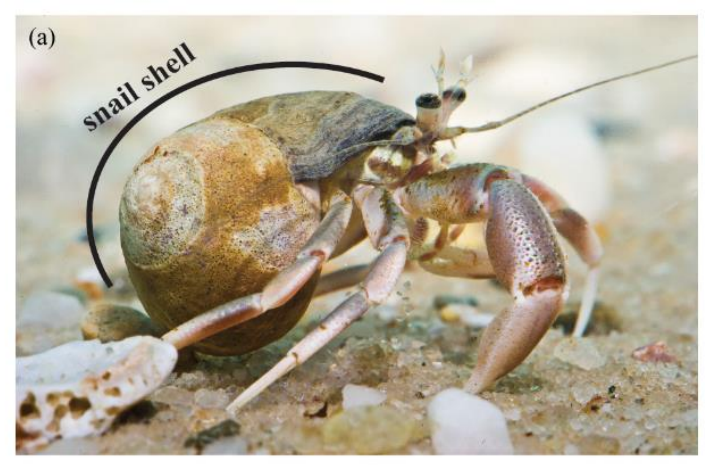

(b)

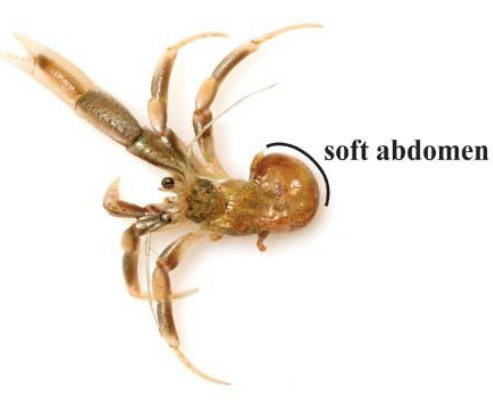

Fig. 1. P. longicarpus hermit crab. (a) The crab in its snail shell. (b) The crab removed from its snail shell to show its soft abdomen. 
Virtually all of the approximately 1100 species of hermit crabs have soft abdomens (Figure 1) and live in and carry around the shells of dead gastropods (snails) as portable shelters for protection against predators and desiccation $[7,8]$. Because hermit crabs grow throughout their lives, they must frequently find new and larger shells. However, most hermit crabs cannot kill snails. Instead, new shells enter a population of crabs when predators, disease, or environmental conditions kill gastropods $[9,10]$. Consequently, new shells are often in short supply, especially those needed by larger individuals $[11,12,13]$.

How could the distribution of wealth in humans compare to the distribution of shells in hermit crabs? One possibility is that the two distributions should be very different. It seems reasonable that human wealth distribution should reflect complex mechanisms based upon culture and social institutions, while the shell size distribution should be driven simply by biological forces such as survival and growth in either the crabs or the gastropods whose shells the crabs occupy. That is, if biological forces determined shell distribution it should reflect either (1) the size distribution of surviving crabs (assuming the proper shell sizes were available from dead snails) or (2) the size distribution of shells from dying gastropods. In other words, according to these two hypotheses, either the biological factors of crab growth or snail deaths should determine the distribution of shell sizes occupied by the crabs.

We have adapted the three classic survivorship patterns from ecology [14] to predict what the shell distributions should be according to these hypotheses. If the first hypothesis were correct, that the shell distribution was determined by crab growth and survival, the distribution could fit any one of the three classic patterns: Type I if all size classes of crabs had a high probability of survival until senescence (Figure 2a), Type II if the crabs had a constant rate of mortality (Figure 2b), or Type III if most of the crabs died at small sizes (Figure 2c). 
In the second hypothesis were correct, that the shell distribution was determined by the survival patterns in the snails, research indicates that the distribution should resemble a Type III pattern. Gosselin and Qian [15] report, for example, that most gastropod species studied have a Type III survivorship pattern (Figure 2c). Due to the very high rate of death in very small snails, the great majority of crabs should be in very small shells with much smaller percentages in shells just a bit larger.

A third hypothesis is that the crab shell distribution takes a form like those commonly reported for humans (Figure 2d). These latter wealth distributions are typically unimodal, rightskewed, and fat-tailed. Perhaps, in contrast to the first two hypotheses, there are some fundamental forces that create similar inequality distributions across species, even in the absence of culture.
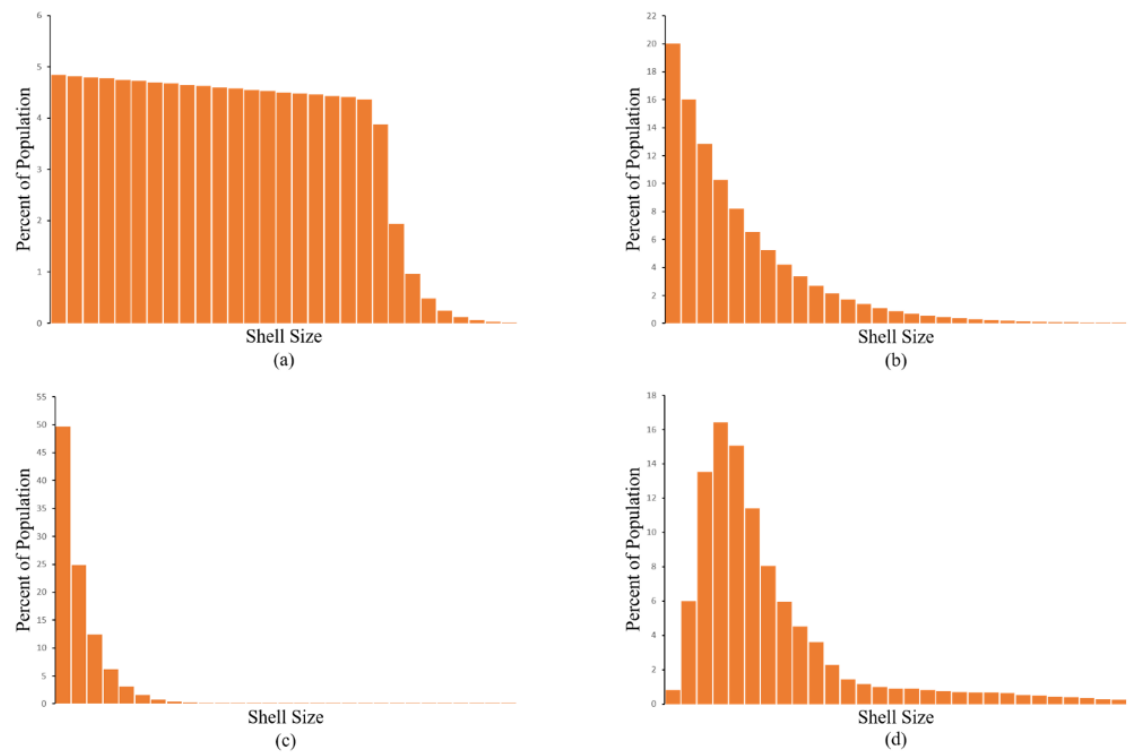

Fig. 2. Four possible distributions of shell sizes occupied by P. longicarpus. Distributions (a)(c) are based on the three classic survivorship curves described in ecology. (a) All individuals 
have a high probability of survival until senescence (Type I curve). (b) Individuals have a constant rate of mortality (Type II curve). (c) Most individuals die at an early age (Type III curve). (d) The typical form of a human wealth distribution. (Note: Curves (a) - (c) are not drawn on log scales as is usual for survivorship curves.)

\section{METHODS}

2.1 Sampling. We studied the distribution of shell sizes in Pagurus longicarpus, a small hermit crab common on the eastern coast of the North America from the Gulf of Mexico to Nova Scotia, Canada. We gathered the crabs $(\mathrm{N}=297)$ on several low tides in a pool approximately $140 \mathrm{~m}$ by $35 \mathrm{~m}$ at West Meadow Beach on Long Island, New York $\left(40^{\circ} 56^{\prime} 41^{\prime} \mathrm{N}, 73^{\circ} 8^{\prime} 41^{\prime \prime} \mathrm{W}\right) . \mathrm{We}$ collected the crabs at random locations within the pool using a $0.5 \times 0.5 \mathrm{~m}$ quadrat and carefully sorted through the sandy substrate to make sure we did not miss crabs in small shells. Because we only sampled in one location, future studies should sample hermit crabs in various locations to assess the generality of our findings.

2.2 Removing Crabs from Their Shells. We removed the hermit crabs from their shells by brief immersion in warm $\left(35^{\circ} \mathrm{C}\right)$ fresh water. We then weighed each crab (damp weight $\pm 0.001 \mathrm{~g}$ ) and measured its carapace length (digital calipers, $\pm 0.01 \mathrm{~mm}$ ). The crabs ranged in weight from $0.003 \mathrm{~g}$ to $0.522 \mathrm{~g}$ and in carapace length from $1.3 \mathrm{~mm}$ to $7.66 \mathrm{~mm}$. After measuring the crabs, we gave each a new shell and returned them to the beach approximately $400 \mathrm{~m}$ from where they were collected.

2.3 Measuring Shells. We dried the shells taken from the crabs at $60^{\circ} \mathrm{C}$ for at least 24 hours and then weighed them, measured their length (maximum dimension along the coiling axis) and width (maximum dimension perpendicular to the coiling axis), and recorded the species and 
condition of each shell. Following many other studies on hermit crabs, we used shell weight as the best measure of resource size for our crabs $[7,12,16]$. Nearly all of the crabs were in Tritia (Ilyanassa) trivittata and Tritia (Ilyanassa) obsoleta shells. Since these species have geometrically similar shells, we used shell weight as a measure of size for both species. The weights of the shells ranged from $0.022 \mathrm{~g}$ to $0.984 \mathrm{~g}$.

2.4 Gini Coefficient. The Gini coefficient measures the inequality of the distribution of a numerical quantity in a given population, for instance, the wealth of individuals or households, or the size of animals. Intuitively, if all individuals in the sample have the same wealth or size, Gini $=0$. If one of them has all of the wealth, and all the others have zero wealth, then Gini $=1-$ $1 / n$ (where $n$ is the population size). In practice, given a series of values $x_{1}, \ldots, x_{n}$ of the quantity in the sample population, the Gini coefficient is given by the following formula [17]:

$$
\text { Gini }=\sum_{i, j=1}^{n}\left|x_{i}-x_{j}\right| /\left(2 n \sum_{i=1}^{n} x_{i}\right)
$$

2.5 Hill Estimation of a Power-Law Exponent. When a sample $\left(x_{1}, \ldots, x_{n}\right)$ is distributed according to a power law, the resulting fat tail prohibits using usual moments of the distribution to estimate parameters. In this case it is preferable to use the Hill estimator to get an estimate of the power law exponent that drives the thickness of the tail [18].

If the sample follows a Pareto law with exponent $\alpha$, the proportion of data points above a given threshold $\theta$ is proportional to $\theta^{\alpha}$. If one rearranges the sample $\left(x_{[1]}, \ldots, x_{[n]}\right)$ in decreasing order (assuming we are estimating the right hand side tail of the distribution), $x_{[1]}$ is the largest element in the sample, $x_{[2]}$ is the second largest, etc. Taking $\theta=x_{[k]}$ for a given value of $k<<n$, 
the number $k$ of samples of data points larger or equal to $\theta$ should be $\lambda \theta^{-\alpha}=\lambda x_{[k]}{ }^{-\alpha}$. Then by taking the logarithm, we get: $\operatorname{Ln}(k)=-\alpha \operatorname{Ln}\left(x_{[k]}\right)+\beta$ with $\beta=\operatorname{Ln}(\lambda)$.

To use the Hill estimator of the power law exponent, one selects a portion $\rho$ of the largest elements of the sample $\left(x_{[1]}, \ldots, x_{[k]}\right)$, with $k=\rho n$ (more precisely the integer part of $\rho n$, typically $\rho=10 \%-15 \%$, with $13 \%$ the value which is used in our calculations). One then performs a linear regression (with constant) of $\left(y_{1}, \ldots, y_{k}\right)$ with $y_{i}=\operatorname{Ln}(i)$ against $\left(\xi_{1}, \ldots, \xi_{k}\right)$ with $\xi_{I}=\operatorname{Ln}\left(x_{[i]}\right)$. Because $y_{i}$ increases whereas $\xi_{I}$ decreases, the slope is negative and the coefficient $\alpha$, which is the negative of the slope, is positive. The intercept $\beta$ is useful to determine the distribution quantiles.

\section{RESULTS}

The distribution of shell sizes that we found in a random sample of 297 crabs (Figure 3) does not resemble any of those distributions suggested by the first two hypotheses (Figure 2a-c). In particular, the actual shell distribution is not like Figure 2c, which is based upon a Type III pattern that is common in many snails and other marine invertebrates [15]. Consequently, the shell distribution does not appear to be a simple reflection of the biological features of either crab or snail growth and survival.

The distribution observed (Figure 3 ) does, however, resemble the one in Figure $2 d$ - the common form of human wealth distributions. Like those distributions, it has a major mode and is skewed to the right with a fat tail. However, the observed distribution also has a secondary mode for shells in the 350 - $399.9 \mathrm{mg}$. range. This mode is due to a deficit in the frequency of shells in 
the 300 to $349.9 \mathrm{mg}$. range. This deficit could have arisen through statistical randomness, or it could be due to causes that are not relevant for understanding the equilibrium distribution.

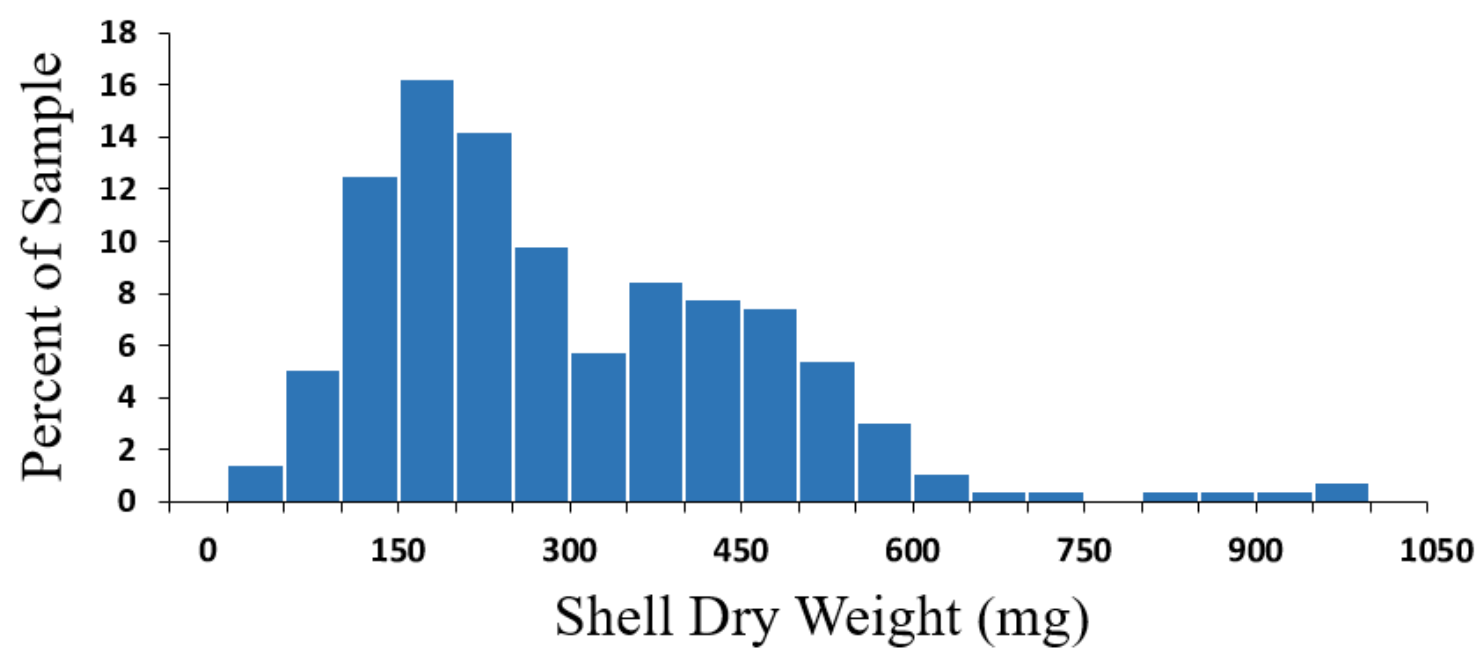

Fig. 3. The observed distribution of shell sizes occupied in P. longicarpus. Shell size is indicated by weight in milligrams. This distribution resembles those commonly found for wealth in human groups.

In general the distribution of shells exhibits a level of inequality more like that in small-scale human groups rather than in large-scale, nation-based ones. First, the Gini coefficient for the crabs is 0.32 (0.02 standard deviation of the estimation of the Gini with two-sided , 5\% confidence interval $=0.285-0.35)$. This is greater than the estimates for some small-scale human groups but less than those for present nation states. For example, Kohler et al. [4] report median Ginis for samples of hunter-gatherers, horticulturalists, and agriculturalists as 0.17, 0.27, and 0.35, respectively, while Borgerhoff Muldler et al. [3] give average Ginis for material wealth in samples for the same kind of groups at $0.36,0.52$, and 0.57 . The Ginis for nation states run from about 0.55 for Japan to 0.80 for the United States [19]. By coincidence, Piketty [14] suggests that the Gini in an "ideal" human society would be 0.33 . 
Second, the extent of the right skew in the shell distribution for the crabs is not as extreme as that in large-scale human societies. In large-scale human societies, some people accrue enormous amounts of wealth compared to the median levels in their groups. This produces a more extreme right tail in the distributions for those societies. For example, in the contemporary United States the richest $1 \%$ of the population owns nearly $40 \%$ of the total wealth [20]. In our sample of hermit crabs, however, there are no Warren Buffets or Jeff Bezoses, and the top $1 \%$ of the crabs only owns about $3.2 \%$ of the total shell weight. However, our sample may somewhat underrepresent the skew of the shell distribution. Just as even a large sample would probably underrepresent the proportion of exceedingly rich people in a human population, our sample may have missed some crabs with very large shells. In fact, there are reports that $P$. longicarpus crabs are capable of occupying larger shells than the ones used by crabs in our sample [21].

Third, the power-law exponent for the shell distribution is considerably smaller than that for many large-scale human societies. Many researchers suggest that the tails of human wealth distributions can be modeled by a power-law or Pareto distribution [22,23]. Power-law distributions are characterized by some quantity raised to a negative power, and the size of that exponent can provide a measure of the fatness of the tail of a wealth distribution. Smaller negative exponents indicate fatter tails and larger negative powers denote thinner tails. Typical estimates for the exponents in the power-law portions of wealth distributions in large-scale, market-based human groups are in the range of a little more than -1.0 to a little under -3.0 . Our estimate of the power-law exponent for the shell distribution is -4.4 (Figure 4). Thus, the right tail of the shell distribution is clearly not as fat as those of large-scale human societies such as 
nations (but see [24] for the problems in estimating power-law exponents in wealth and other distributions).

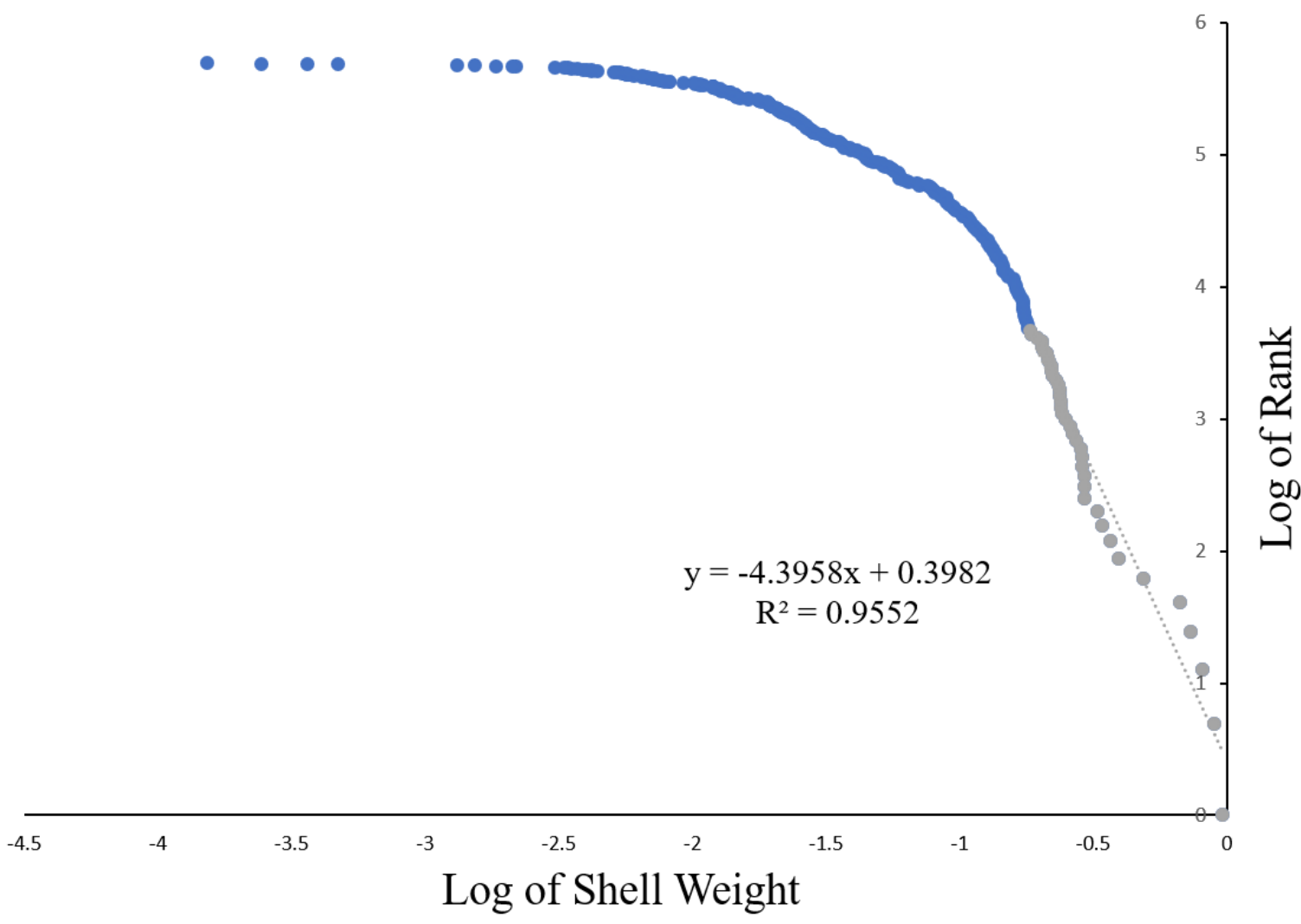

Fig. 4. Hill estimation of the power-law exponent for the shell distribution. The exponent is given by the slope of the log-log regression of the ranks of the shells versus their size. The regression is for the 39 largest shells in the sample. For a sample of $n=39$, given the slight bias of this estimator for small samples, an unbiased estimate of the shape parameter is around -4.7, while its uncertainty (standard deviation of the error) is $\alpha \sqrt{2 / \mathrm{n}}=0.98$.

\section{DISCUSSION}


Our results indicate that the distribution of shells occupied in P. longicarpus is generally similar in form to distributions of wealth in human societies, especially those in small-scale human groups. What could cause this similarity? One possibility is that the resemblance could be purely coincidental. Many different mechanisms can produce unimodal distributions skewed to the right with fat tails. Consequently, the two distributions could be generated by entirely different kinds of mechanisms. By the same token, it is also possible that the likeness of wealth distributions in different human groups is also coincidental and generated by different mechanisms, say, for example, in hunter-gatherers and developed nations.

A second possibility is that analogous factors in both hermit crabs and humans generate the similarity in their inequality distributions. If this were the case, those analogous factors would have to be of the most basic sort since, as noted above, hermit crabs are so different in most respects from human beings.

What could those comparable factors be? Researchers have suggested two possibilities for explaining the forms of human inequality distributions that we propose might also work for hermit crabs: individual differences and resource transfer. Approaches using individual differences to account for human inequality distributions, such as human capital theory and a variety of mathematical models, assume that people gain wealth in relation to their talents (such as training, education, and personality) plus, in some cases, random events [25,26,27,28]. In contrast, the intergenerational property transfer approach assumes that the form of the wealth distribution is strongly influenced by bequests and gifts from one generation to another $[3,27,29,30,31,32]$. A bequest is a property transfer made to one individual upon the death of another, and a gift is an allocation from one living individual to another. 
Researchers attempt to evaluate these two approaches by building models based upon them and then comparing the wealth distributions thus generated with wealth distributions derived from empirical studies. Some of the liabilities of this reverse engineering, or attempt at inverse modelling, of social hierarchies are discussed in Chase and Lindquist [33]. However, we suggest that the core assumptions of these two approaches on the form of inequality distributions can be experimentally evaluated by using hermit crabs. This would allow us to directly observe the dynamics actually generating inequality systems.

In hermit crabs there is no deliberate intergenerational transfer of property. However, crabs do transfer gastropod shells to one another through vacancy chain processes [16], and experimenters can readily observe these processes under laboratory conditions. In a vacancy chain, a new resource unit coming into a population sets off a series of transfers among several individuals allowing them to obtain new and usually larger units. For example, in hermit crabs, when a snail dies, its shell may be taken by a first crab that leaves its old and usually smaller shell behind. A second crab will then occupy the abandoned shell left by the first crab and desert its previous, smaller shell, and so on. In humans, vacancy chains occur in the distribution of houses, jobs, and cars [34,35,36,37].

A possible experiment to evaluate the importance of property transfers on inequality in the distribution of snail shells in hermit crabs is as follows: An experimenter assembles a large number of groups of hermit crabs all having the same-sized, small shells. The experimenter periodically gives each group of crabs new, empty snail shells of the same size on an identical schedule. However, the experimenter allows vacancy chains to proceed in half of the groups (the treatment groups), but not in the other half (the comparison groups). For the comparison groups, the experimenter removes the old shells left behind by the crabs obtaining new shells, prohibiting 
shell transfers from one crab to another. In the treatment groups, allowed to use vacancy chains, the experimenter does not remove the old shells abandoned by those crabs taking the initial shells, allowing shell transfers among crabs. Previous research has shown that about three crabs get new snail shells in a vacancy chain: one crab gets the initial shell and two get shells via transfers [16]. If property transfers are an important factor in generating the form of the shell inequality distribution we observed for the crabs we collected (Figure 3), we would expect that unimodal, right-skewed, and fat-tailed distributions would emerge over time in the group allowed to have vacancy chains, but not in the comparison groups.

\section{CONCLUSIONS}

The distribution of gastropod shell sizes occupied in P. longicarpus is similar in form to wealth distributions in many human societies. In particular, the characteristics of the shell distribution (Gini coefficient, extent of skew, and power-law exponent) are more in line with smaller-scale human populations such as those found in earlier human civilizations and groups such as hunter-gathers and horticulturalists. This finding suggests the possibility that some fundamental factors create similar inequality distributions across species. The crab shell distribution does not seem to represent simple differences in either hermit crab or gastropod growth and survival rates. Additionally, hermit crabs might be useful as an animal model to experimentally investigate the importance of various factors on the generation of inequality. Investigations of this sort would allow us to harness the power of the experimental approach to provide greater understanding of how inequality systems work.

Finally, our results demonstrate that, in a manner of speaking, inequality is "natural". That is, inequality can occur in groups in which individuals own material resources but are neither human nor subject to formal social practices or cultural traditions. As such, hermit crabs 
can provide a kind of reference point with which to compare inequality in both smaller- and larger-scale human societies.

\section{REFERENCES}

[1] Flannery, K. \& Marcus, J. The Creation of Inequality. (Harvard University Press, 2014).

[2] Piketty, T. Capital in the twenty-first century. (Harvard University Press, 2014).

[3] Borgerhoff Mulder, M. et al. Intergenerational wealth transmission and the dynamics of inequality in small-scale societies. Science 326, 682-688 (2009).

[4] Kohler, T. A. et al. Greater post-Neolithic wealth disparities in Eurasia than in North America and Mesoamerica. Nature 551, 619-622 (2017).

[5] Strassmann, J. E.\& Queller, D.C. Privatization and property in biology. Animal Behaviour 92, 305-311 (2014).

[6] Scheffer, M., van Bavel, B., van de Leemput, I. A. \& van Nes, E. H. Inequality in nature and society. Proceedings of the National Academy of Sciences, 114, 13154-13157 (2017).

[7] Reese, E. S. The behavioral mechanisms underlying shell selection by hermit crabs. Behaviour 21, 78-126 (1963).

[8] Vance, R. R. The role of shell adequacy in behavioral interactions involving hermit crabs. Ecology 53, 1075-1083 (1972b).

[9] Wilber, T. P. \& Herrnkind, W. Rate of new shell acquisition by hermit crabs in a salt marsh habitat. Journal of Crustacean Biology 2, 588-592 (1982).

[10] Wilber, T. P. \& Herrnkind, W.F. Predaceous gastropods regulate new-shell supply to salt marsh hermit crabs. Marine Biology 79, 145-150 (1984).

[11] Vance, R. R. Competition and mechanism of coexistence in three sympatric species of intertidal hermit crabs. Ecology 53, 1062-1074 (1972a).

[12] Bach, C., Hazlett, B. \& Rittschoff, D. Effect of interspecific competition on fitness of the hermit crab Clibanarius tricolor. Ecology 57, 579-586 (1976).

[13] Kellogg, C. W. Gastropod shells: a potentially limiting resource for hermit crabs. Journal of Experimental Marine Biology and Ecology 22, 101-111 (1976).

[14] Demetrius, L. Adaptive value, entropy, and survivorship curves. Nature 275, 213-214 (1978). 
[15] Gosselin, L. A. \& Qian, P.Y. Juvenile mortality in benthic marine invertebrates. Marine Ecology Progress Series 146, 265-282 (1997).

[16] Chase, I. D. Vacancy chains. Annu. Rev. Sociol. 17, 133-154 (1991).

[17] Gini, C. Concentration and dependency ratios. Rivista di Politica Economica 87, 769-789 (1997).

[18] Hill, B. M. A simple general approach to inference about the tail of a distribution. The Annals of Statictics 3, 1163-1174 (1975).

[19] Davies, J. B., Sandström, S., Shorrocks, A. \& Wolff, E.N. The level and distribution of global household wealth. The Economic Journal 121, 223-254 (2011).

[20] Bricker, J. D. et al. Changes in US family finances from 2013 - 2016: evidence from the survey of consumer finances. Fed. Res. Bull. 103, 1-42 (2017).

[21] Weissburg, M., Roseman, L., Chase, I. D. Chains of opportunity: a Markov model for acquisition of reusable resources. Evolutionary Ecology 5, 105-117 (1991).

[22] Pareto, V. Cours d'économic politique. (Droz, 1896).

[23] Drăgulescu, A. \& Yakovenko, V. M. Exponential and power-law probability distributions of wealth and income in the United Kingdom and the United States. Physica A: Statistical Mechanics and Its Applications 299, 213-221 (2001).

[24] Clauset, A., Shalizi, C. R. \& Newman, M. E. Power-law distributions in empirical data. SIAM Review 51, 661-703 (2009).

[25] Mincer, J. Investment in human capital and personal income distribution. Journal of Political Economy 66, 281-302 (1958).

[26] Becker, G. S. Human capital. (University of Chicago Press, 1964).

[27] Cagetti, M. \& De Nardi, M. Wealth inequality: Data and models. Macroeconomic Dynamics 12, 285-313 (2008).

[28] Pluchino, A., Biondo, A. \& Rapisarda, A. Talent vs Luck: the role of randomness in success and failure. arXiv preprint arXiv:1802.07068 (2018).

[29] Becker, G. S. \& Tomes, N. An equilibrium theory of the distribution of income and intergenerational mobility. Journal of Political Economy 87, 1153-1189 (1979).

[30] Gale, W. G. \& Scholz, J. K. Intergenerational transfers and the accumulation of wealth. Journal of Economic Perspectives 8, 145-160 (1994). 
[31] Kotlikoff, L. J. \& Summers, L. H. The role of intergenerational transfers in aggregate capital accumulation. Journal of Political Economy 89, 706-732 (1981).

[32] Arrow, K. J. \& Levin, S. A. Intergenerational resource transfers with random offspring numbers. Proceedings of the National Academy of Sciences 106, 13702-13706 (2009).

[33] Chase, I.D. \& Lindquist, W.B. The fragilty of individual-based explaqnations of social hierarchies: a test using animal pecking orders. PLOS One, (2016).

[34] Lansing, J., Clifton, C. \& Morgan, J. New homes and poor people: a study of chains of moves. (University of Michigan Institute for Social Research, 1969).

[35] White, H. C. Chains of Opportunity: System Models of Mobility in Organizations. (Harvard University Press, 1971).

[36] Pinfield, L. T. The Operation of Internal Labor Markets. (Springer, 1995).

[37] Smith, T. H. The Marketing of Used Automobiles. (Ohio State University Press, 1941).

Acknowledgements We thank Stephen Abrams, Mark Aronoff, Mark Granovetter, Lawrence Martin, Axel Meyer, Everett Waters, Nicholas Wilson, and Lydia Zepeda for comments; Moez Ahmed, Alyson Hall, Abigail Higgins, Eva Muchnick, and Derek Ng for help with data collection; Alyson Hall for data organization and analysis; Paul St. Dennis, Jason Jones, and David Charifson for technical assistance; Lester Lefkowitz for photographs; and Ayush Kumar and Jiwon Kim for figures. The comments of three anonymous reviewers greatly improved this paper.

Author Contributions I.D.C. and D.K.P. conceived the project; I.D.C. and D.K.P. collected the data; R.D. analyzed the data; all authors wrote the paper; and all authors contributed to the interpretation of the results and commented on the final draft on the manuscript.

Funding: This research did not receive any specific grant from funding agencies in the public, commercial, or not-for-profit sectors. 
Declarations of Interest: None. 